\title{
Gamma spectroscopy of nuclei far from stability
}

\author{
O. Sorlin
}

Institut de Physique Nucléaire, IN2P3-CNRS, F-91406 Orsay, France

The focus of the present review is the study of neutron-rich medium-mass nuclei around major shells or subshells $\mathrm{N}=20,28$ or 40 . Coulomb excitation of secondary beams and in-beam spectroscopy study using the fragmentation of a stable beam have brought a wealth of informations concerning the behaviour of the closed shells when nuclei contain large neutron excesses. Pionneering experiments in this field are presented with special emphasis on the work achieved at the GANIL accelerator.

\section{INTRODUCTION}

From the study of stable nuclei over the last decades, it has been demonstrated experimentally that some edifices of nucleons are most tightly bound than others. The extra-binding energy of these nuclei occurs for nuclei comprising a magic number of protons $(\mathrm{Z})$ or/and neutrons $(\mathrm{N})$. This feature looks a priori similar to atomic systems where rare gas atoms exhibit a higher ionization potential than the neighbouring elements. If both atoms and nuclei can be modelized by mean field approaches, the specificity of nuclear systems should be pointed out. In nuclei, the mean field is self-generated by the nucleons, whereas in atoms, the mean field is generated by the long-range interaction between the electrons of the atomic cloud and the protons of the nucleus. Also, nuclei contain two fluids of quantum particles and their respective excesses (proton or neutron) could provide a profound modification of the nuclear potential. It is for instance expected that the surface of very neutron-rich nuclei would be essentially composed with a diffuse neutron-matter [1]. Contrary to the protons which are sensitive to the Coulomb barrier and therefore cannot extend far from the nucleus core, neutrons can develop halos or skins which modifies the density at the surface of the nucleus. This would result in a change of the mean field potentials as compared to those defined for nuclei in the valley of stability, a vanishing of the major spherical magic closed-shells, and subsequent appearance of new magic numbers. However, evidences of this new phenomenon are often difficult to obtain since an "apparent" reduction of shell gaps could be due to the deformation of the nucleus. In such case, different magnetic substates of a given orbitals have not equal energy, those along the axis of deformation being more tightly bound to the nucleus. As a consequence, the spherical single particle energies are getting closer to each others, after the splitting of the Nilsson orbitals as a function of the deformation. Energy gaps can appear from this new orbital configurations at large deformation parameter. For some neutron-rich nuclei close or at major shells $\mathrm{N}=20, \mathrm{~N}=28$, strongly deformed shapes are predicted to be separated from spherical one by energies of few hundred keV or less. In such cases, it is not easy to predict what would be the shape of the ground state configu- 
ration, minima of the potential energy being often very shallow and model dependant [2]. This situation of strong mixing between different shapes of a given nucleus, the so-called shape co-existence, is unique in quantum physics systems. If this has already been evidenced in heavy nuclei, it has not been proven yet for medium light exotic-nuclei. One

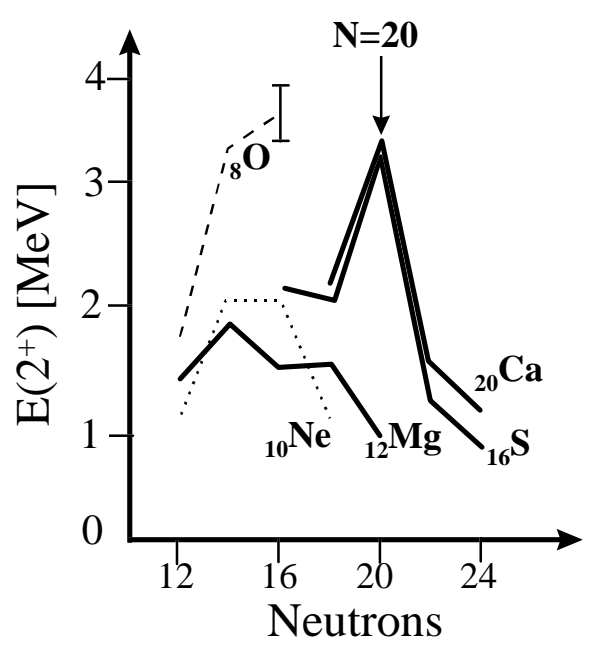

Figure $1.2^{+}$-energies in the $\mathrm{Ca}, \mathrm{S}, \mathrm{Mg}, \mathrm{Ne}$, and $\mathrm{O}$ isotopic chains.

of the goal of nuclear spectroscopy with exotic nuclei will be to search for a modification of the spherical mean-field provided by the excess of proton or neutrons. This has to be preferentially investigated with Z-magic nuclei, as $\mathrm{O}, \mathrm{Ca}, \mathrm{Ni}, \mathrm{Sn}$, which are expected to be spherical in their ground state configuration. With this requirement, it may be possible to isolate the effect of mean-field modification from changes in the level-energies induced by deformation. For very exotic species, nuclear structure informations should be extracted from "easy to measure" quantities. For even-even nuclei, first $2^{+}$excited state, excitation probability $B\left(E 2: 0^{+} \rightarrow 2^{+}\right)$, and $4^{+} / 2^{+}$energy ratios provide valuable information about the shape of a nucleus, and rigidity against excitations. Complementary information is provided, for even-odd or odd-even nuclei, by the determination of the energy of the single particle orbitals across the spherical gap deduced from their level scheme. The next section presents a selection of important experimental results obtained at GANIL around closed shells $\mathrm{N}=20,28$ and 40 .

\section{SELECTED EXAMPLES}

\subsection{Neutron-rich light nuclei}

First indications of the vanishing of a shell strength have been revealed around the neutron number $\mathrm{N}=20$. It was found that ${ }^{31} \mathrm{Na}$ and ${ }^{32} \mathrm{Mg}$ present anomalies in their binding energies [3], mean-square radii [4] and nuclear spectra [5,6]. Figure 1 shows the $2^{+}$-energies of nuclei $\mathrm{N}=20$ nuclei exhibiting different $\mathrm{N} / \mathrm{Z}$ ratios. It is seen that the $2^{+}$ energies are increased for $\mathrm{Ca}$ and $\mathrm{S}$ nuclei when reaching $\mathrm{N}=20$, as expected for magic 


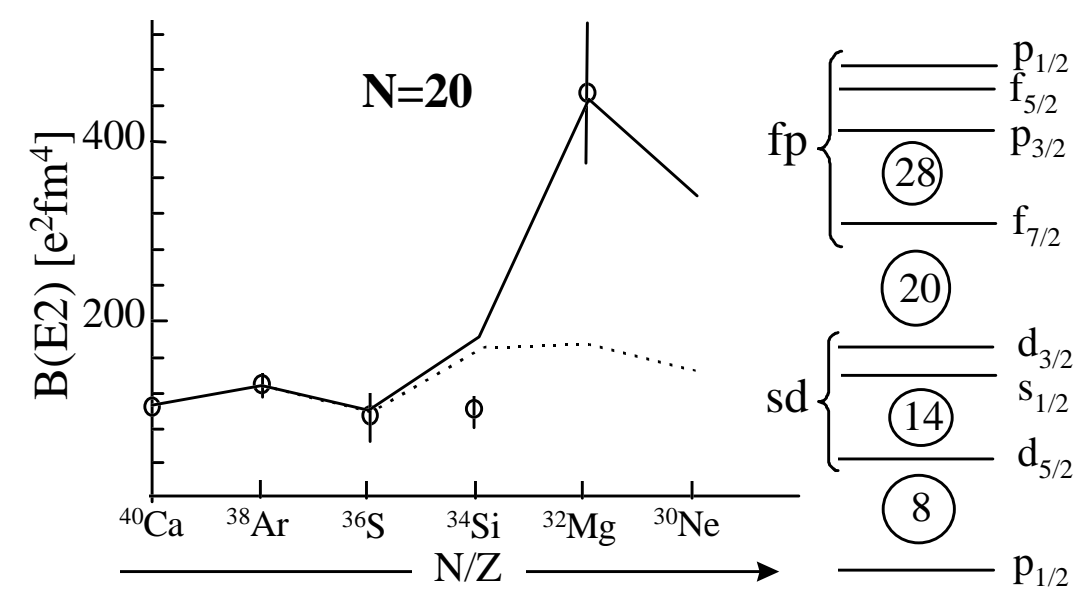

Figure 2. Measured $\mathrm{B}(\mathrm{E} 2)$ values as a function of neutron-richness for $\mathrm{N}=20$ isotones. Shell-model calculations using sd or $\mathrm{sd}+\mathrm{fp}$ valence space are shown with dotted and full line, respectively. [8]. Schematic ordering of the single particle levels are shown on the right part.

nuclei in which excitations occur across a large energy gap. The removal of four protons from ${ }^{40} \mathrm{Ca}$ has not dramatically modified the structure of the nucleus, the $2^{+}$energy of ${ }^{36} \mathrm{~S}$ being very similar to that of ${ }^{40} \mathrm{Ca}$. The $\mathrm{B}(\mathrm{E} 2)$ partly reflects the possibility of making $2^{+}$excitations from the ground state configuration with particle(s)-hole(s) (ph) excitations. Nuclei with filled shells, as ${ }^{40} \mathrm{Ca}$, have a very small $\mathrm{B}(\mathrm{E} 2)$ since ph-excitations within sd valence-space are blocked. They have to occur across the $\mathrm{N}=20$ shell gap and therefore proceed only via small matrix elements. These magic nuclei are coined as very "rigid" or weakly collective. While decreasing the proton number, it is expected that the vacant holes would increase the possibility of making ph-excitations. Fig. 2 shows that the $\mathrm{B}(\mathrm{E} 2)$ remains remarkably constant from ${ }^{40} \mathrm{Ca}$ to ${ }^{34} \mathrm{Si}[7]$. When renormalized to Weisskopf units (W.u.), in order to take into account the "liquid drop" dependance in $A^{4 / 3}$, the $\mathrm{B}$ (E2,W.u.) is increased by about only $20 \%$ from ${ }^{40} \mathrm{Ca}$ to ${ }^{34} \mathrm{Si}$. This trend is a priori unexpected since the possibility of making $2^{+}$excitations with particle-hole excitations and hereafter the $\mathrm{B}(\mathrm{E} 2)$ should be at its maximum value at mid sd-shell $(\mathrm{Z}=14)$ since the number of particles and holes is at its maximum value. This feature points towards a strong subshell gap at $\mathrm{Z}=14$. It is however important to notice that, due to the low level densities of these light nuclei, each shell filling may result in a shell closure behaviour. The $\mathrm{Z}, \mathrm{N}=16$ is therefore naturally expected to exhibit a similar effect, though weaker, with the filling of the $\mathrm{s}_{1 / 2}$ level.

A drastic change in the $2^{+}$-energy and $\mathrm{B}(\mathrm{E} 2)$ patterns is seen in ${ }^{32} \mathrm{Mg}$, while removing again four protons from ${ }^{36} \mathrm{~S}$. The large collectivity of ${ }^{32} \mathrm{Mg}, \mathrm{B}(\mathrm{E2})=454(78) \mathrm{e}^{2} \mathrm{fm} \mathrm{m}^{4}$, has been first determined by Motobayashi et al. [8] by coulomb excitation. Confirmation of a large $\mathrm{B}(\mathrm{E} 2)$ is found in other references $[9,10]$. This abnormal increase of collectivity in 
${ }^{32} \mathrm{Mg}$ indicates a clear structural change of the nucleus. Shell model calculations taking into account either sd states (dotted line in Fig. 2), or a wider valence space $\mathrm{sd}+\mathrm{fp}$ states (full line) show the importance of intruder states in the modelization of ${ }^{32} \mathrm{Mg}$. From these predictions, it is expected that the role of the $\mathrm{fp}$ intruder states is maximum in ${ }^{32} \mathrm{Mg}$ and progressively reduced in ${ }^{30} \mathrm{Ne}$ and in ${ }^{28} \mathrm{O}$. The concept of intruder orbitals can be viewed within the deformed mean field picture through the presence of a high density of states at large deformation originating from the energy splitting of shells. Far now, no spectroscopic information is available for ${ }^{30} \mathrm{Ne}$, which is hitherto too weakly produced. Nevertheless, the technique of in-beam spectroscopy using the projectile fragmentation of a ${ }^{36}$ beam at an energy of $77 \mathrm{~A} . \mathrm{MeV}$ onto a $2.76 \mathrm{mg} . \mathrm{cm}^{-2}$ Be target has brought new spectroscopic informations on neutron-rich nuclei as ${ }^{22} \mathrm{O},{ }^{26-28} \mathrm{Ne}$ and ${ }^{32} \mathrm{Mg}[11,12]$. The fragments produced in the collision were selected by the SPEG spectrometer at GANIL. A highly efficient ( $25 \%$ at $1.33 \mathrm{MeV}$ ) gamma array was used to detect the photons arising from the de-excitation of the fragments in flight. It was composed with $74 \mathrm{BaF}_{2}$ crystals installed symmetrically at the upper and lower hemisphere around the target. Four Ge detectors were installed in between the two hemispheres in order to help identifying closeenergy peaks in the $\gamma$-spectra which cannot be separated in the $\mathrm{BaF}_{2}$ detectors due to their rather poor intrinsic energy-resolution (about $200 \mathrm{keV}$ after having included Doppler shift broadening). This array was used to establish level schemes of nuclei transmitted via fragment- $\gamma$ coincidences as well as fragment- $\gamma-\gamma$ coincidences. The $2^{+}$-energy of ${ }^{28} \mathrm{Ne}$ (Fig. 3) has been found to be $E\left(2^{+}\right)=1.32 \mathrm{MeV}[11,12]$. The sudden decrease in energy, as compared to ${ }^{26} \mathrm{Ne}$ whose $E\left(2^{+}\right)=2.02(2) \mathrm{MeV}$ [13], indicates that the shell closure at $\mathrm{N}=20$ for $\mathrm{Ne}$ isotopes is probably weak. This is confirmed by the fact that a large valence space $(\mathrm{sd}+\mathrm{fp})$ is necessary to modelize the experimental $2^{+}, 4^{+}$-energies of ${ }^{28} \mathrm{Ne}$ (Fig. 3).

The ideal case to study the modification of the spherical mean-field potential, would be the oxygen isotopic chain. Oxygen isotopes are also magic in proton number, with a large energy gap across $\mathrm{Z}=8$. Their potential energy is expected to be minimum at a spherical shape, with a very steep increase as a function of the deformation parameter of the nucleus. This proton magicity hampers the nuclei to deform, and thus would allow to disentangle the two effects of deformation and mean-field potential change at large $\mathrm{N} / \mathrm{Z}$. Unfortunately, it is found that the drip-line is occuring very "close" to stability, as indicated by the particle unstability of ${ }^{26} \mathrm{O}$ and ${ }^{28} \mathrm{O}[14,15]$. It is however interesting to understand this feature, and why ${ }^{24} O$ is the last bound oxygen. Experimental informations have been recently obtained for ${ }^{22} \mathrm{O}$ and ${ }^{23,25} \mathrm{~F}$ [11] using the in-beam fragmentation of a ${ }^{36} \mathrm{~S}$ beam. For ${ }^{22} \mathrm{O}$, a high $2^{+}$-energy, $E\left(2^{+}\right)=3.19 \mathrm{MeV}$ (Fig. 4) indicates that this nucleus can be considered as a good core to modelize adjacent nuclei. This originates from the strong $\mathrm{N}=14$ subshell as in the valence mirror nucleus ${ }^{34} \mathrm{~S} i$ mentionned before. This high $2^{+}$-energy is associated with a low $\mathrm{B}(\mathrm{E} 2)$ [16], as expected for rigid nuclei. The spectrum of ${ }^{23} F$ (Fig. 4) shows two lines at 910 and $2900 \mathrm{keV}$ which are interpreted as due to the in-beam decay of the $9 / 2^{+}$and $7 / 2^{+}$states respectively. In the fragmentation reaction, Yrast states are mainly populated $[11,17]$. Hence, levels with the highest spin at a given excitation energy as $9 / 2^{+}$and $7 / 2^{+}$are favoured experimentally. The energies of these states $(3810$ and $2900 \mathrm{keV}$ ) are in remarkable agreement with the calculation of Brown et al.[18] (3758 and $2916 \mathrm{keV}$ ). These states belong to the $3 / 2^{+} \ldots 9 / 2^{+}$multiplet, which originates from the coupling of a proton in $\mathrm{d}_{5 / 2}$ to the $2^{+}$core excitation of ${ }^{22} \mathrm{O}$ 



Figure 3. $\gamma$-spectra of ${ }^{26} \mathrm{Ne}$ (with $4 \mathrm{Ge}$ detectors) and ${ }^{28} \mathrm{Ne}$ (with $74 \mathrm{BaF} 2$ detectors). Deduced experimental levels schemes are shown beneath with the calculated spectrum of Brown et al. [18] and Utsono et al. [19] using an sd and sd-fp valence space, respectively.

at $3.19 \mathrm{MeV}$. This suggests that the proton added to ${ }^{22} \mathrm{O}$ is "spectator", and doesn't modify the structure of the core. When interpreting the experimental spectrum of ${ }^{25} F$ in comparisson with Brown's calculations, we can deduce that ${ }^{25} \mathrm{~F}$ can be modelized in the same way as ${ }^{23} \mathrm{~F}$ with a core of ${ }^{24} \mathrm{O}$ whose $2^{+}$energy would lie around $3.5 \mathrm{MeV}$. This increase in energy at $\mathrm{N}=16$ is in accordance with an increased shell effect as suggested by Ozawa et al. [20] by looking at the neutron separation energies systematics of the nuclei with increasing $T_{Z}$. This could suggest a change in the structure of the nuclei connected with the reduction of the $\mathrm{N}=20$ shell gap as predicted by Utsono et al. [19] (see also the proceedings of T. Otsuka and I. Tanihata in the conference). The determination of the $2^{+}$ energy of ${ }^{24} O$, not measured so far, would help us elucidating why the drip line is so close to stability in $\mathrm{O}$ chain. Part of the explanation could reside in the steepness of the potential energy surfaces of oxygen isotopes about spherical shape. Nuclei have subsequently no mean to minimize their potential energy while deforming. This could also be due to a 
reduction of the $\mathrm{N}=20$ shell-gap in ${ }^{28} \mathrm{O}$. This reduction of shell-gap while increasing the $\mathrm{N} / \mathrm{Z}$ ratio is predicted by Utsuno et al [19]. It is noticebale that the addition of a single proton in ${ }^{24} O$ can bind fluorine chain with the addition 6 neutrons, ${ }^{31} F$ [15] being still bound. The study of neutron-rich ${ }^{27,29} \mathrm{~F}$ isotopes would help to understand how and when this extra-proton modifies the spherical Oxygen core. So far, a substantial reduction of the $\mathrm{N}=20$ shell-gap has not been undoubtedly proved or rejected experimentally. This will be one of the challenges for future experiments. The modification of the shell closures for weakly bound light nuclei is connected to the fact that pairing effects, or coupling of the weakly bound states to the continuum are limiting the use of the mean field concept itself in which nucleons can be treated independently [22].
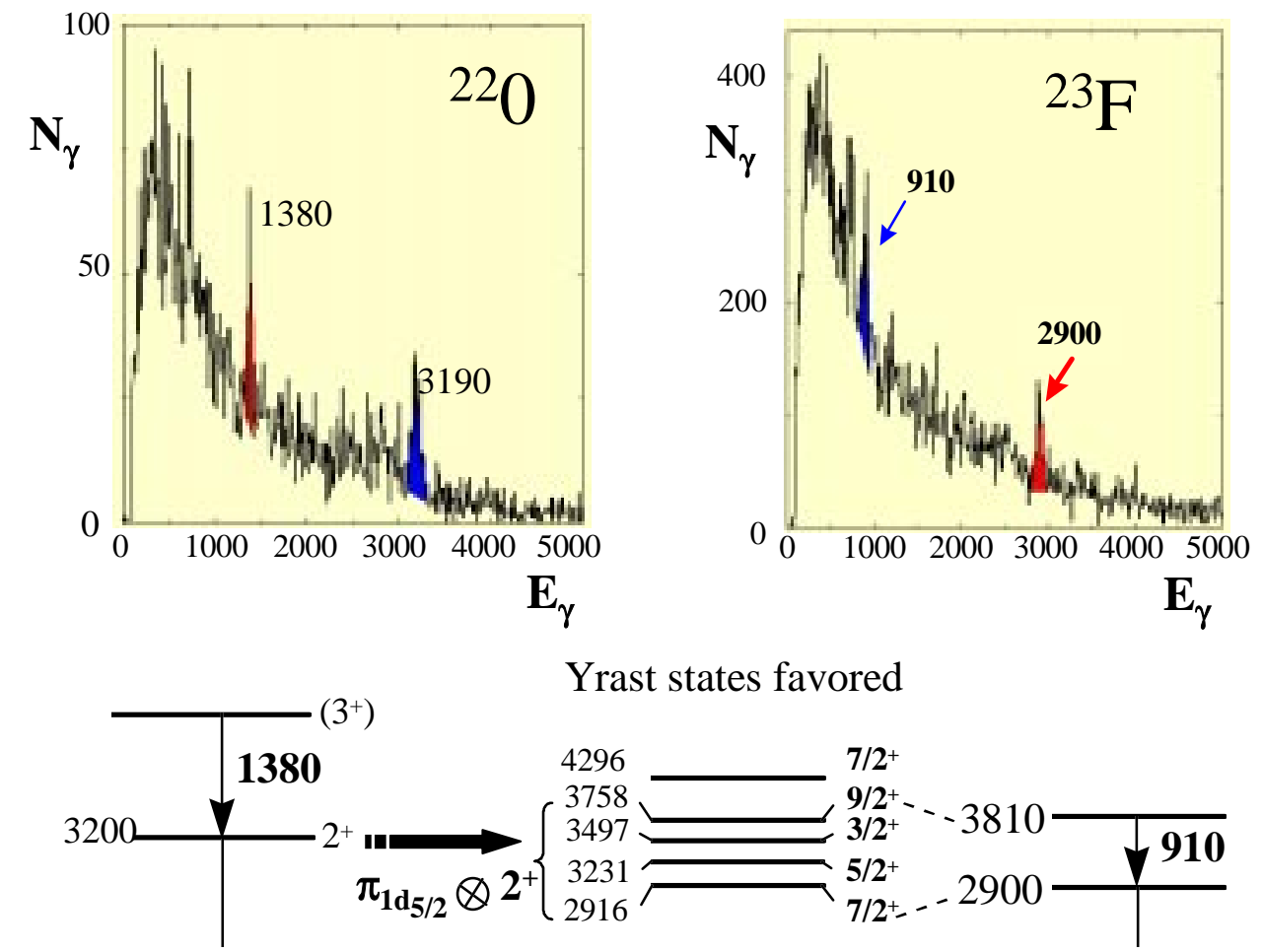

Yrast states favored

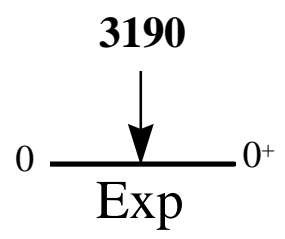

${ }^{22} \mathbf{O}$

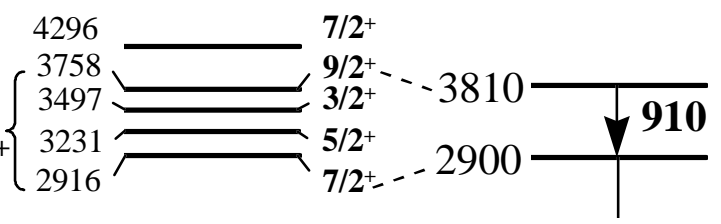

1776



$1 / 2^{+}$

$5 / 2^{+}$

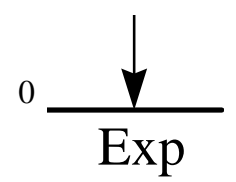

${ }^{23} \mathbf{F}$

Figure 4. $\gamma$-spectra of ${ }^{22} \mathrm{O}$ and ${ }^{23} \mathrm{~F}$. Deduced experimental levels schemes are shown beneath with the calculated spectrum of Brown et al. [18] using an sd-shell valence space. 


\subsection{The $\mathrm{N}=28$ shell-closure}

The study of the $\mathrm{N}=28$ isotones far from stability has been motivated by several points: (i) another test region for shell disappearance as in $\mathrm{N}=20$, (ii) the possible reducing of the spin-orbit strength with increasing isospin, (iii) the understanding of the abnormal ${ }^{48} \mathrm{Ca} /{ }^{46} \mathrm{Ca}$ abundance ratio in the solar system or in refractory inclusions of meteorites. Experimentally, $\beta$-decay properties of $\mathrm{N}=28$ nuclei as ${ }^{45} \mathrm{Cl},{ }^{44} \mathrm{~S}$ and ${ }^{43} \mathrm{P}$ have been determined $[23,24]$. These half-lives $\left(\mathrm{T}_{1 / 2}\right)$ were found to be 3-10 times shorter than expected by Möller et al. [25] who expected spherical shapes for these semi-magic nuclei. Based on a comparisson between QRPA calculations of $T_{1 / 2}$ as a function of the deformation parameter of the nuclei and the measured $\mathrm{T}_{1 / 2}$, it was concluded that ${ }^{44} S$ should be strongly deformed [23], either with a prolate or oblate shape. With the new mass-determination



Figure 5. Experimental and theoretical level schemes of ${ }^{46} A r$ and ${ }^{40-44} S$. Shell model calculations are taken from Nowacki et al. [32]

of ${ }^{44} S$ [26], a deformation of $\beta_{2}=0.30$ has been deduced [27] using the same procedure. Relativistic mean-field calculations of Werner et al. [28] predict a rather complicated situation for ${ }^{44} S$, with nearly degenerate prolate $\left(\beta_{2}=0.25\right)$ and oblate $\left(\beta_{2}=-0.24\right)$ solutions. Reinhard et al. [2] has calculated potential energy surfaces of ${ }^{40,42,44} S$ as a function of $\beta_{2}$ deformation parameter using a large choice of Skyrme forces. The two oblate and prolate 
minima are also found to be close in energy, most of the interactions predicting a prolate ground state with $\beta_{2}$ close to 0.28 for ${ }^{44} S$. These deformation parameter are close to the value $\beta_{2}=0.258(36)$, derived from the measurement of $\mathrm{B}(\mathrm{E} 2)$ by Glasmacher et al. [29] using the prescription of Raman et al. [30] for deformed nuclei. They however conclude that the collectivity of ${ }^{44} S$ rather originates from vibrations [29].

Coulomb excitation of ${ }^{43} S$ has shown a large B(E2) connecting the g.s. to a level at $940 \mathrm{keV}$ [31]. It is not conclusive from their experiment what is the shape of the ground state. Sarazin et al. [26] has evidenced the existence an E2 isomer at $319 \mathrm{keV}$ in the same nucleus, which is interpreted, with the help of shell model calculations as a shape isomer.

In order to obtain more experimental informations on the sulfur isotopes, the search for higher energy states has been undertaken with the in-beam fragmentation of a ${ }^{48} \mathrm{Ca}$ beam [17]. The experimental setting of this experiment was very similar to that described in section 2.1 for the study of $\mathrm{O}, \mathrm{F}$ and Ne isotopes. However, segmented clover have replaced the normal Ge detectors, leading to a substancial increase in efficiency and a better determination of the angle of emission of the $\gamma$ 's emitted in flight after the fragmentation reaction. Level schemes of ${ }^{40,42,44} \mathrm{~S}$ and ${ }^{46} \mathrm{Ar}$, deduced from experimental $\gamma$-spectra are shown in Fig. 5. The ratios of $4^{+} / 2^{+}$energies has been tentatively deduced from this study for ${ }^{40} S$ and ${ }^{42} S$. It is found that the ${ }^{42} S$ exhibits the largest $4^{+} / 2^{+}$energy ratio of 3.08 among all measured sulfur. This ratio is close to the value of 3.3 for a perfect rotor. Shell model calculations [32] are in good agreement both with the experimental values of $2^{+}, 4^{+}$-energies [17] and $\mathrm{B}(\mathrm{E} 2)$ values of ${ }^{40,42,44} S$ [33,29]. They conclude to an erosion of the $\mathrm{N}=28$ gap in the sulfur isotopes, with a maximum of deformation in ${ }^{42} S$. In ${ }^{44} S$, an experimental indication of a $0_{2}^{+}$state at $1615 \mathrm{keV}$ is found [17] through the observation of a $285 \mathrm{keV}$ line which probably populates the $2^{+}$state at $1.33 \mathrm{MeV}$. The energy of this $0_{2}^{+}$at $1.615 \mathrm{MeV}$ is in good accordance with the shell-model calculations [34], $\mathrm{E}\left(0_{2}^{+}\right)=1.5 \mathrm{MeV}$, and slightly higher than the new ones [32]. Further experimental studies has to be achieved in order to prove unambiguously this shape co-existence phenomenon, through the detection of $\gamma$-lines connecting different shapes.

\subsection{The Ni isotopic chain, a terrestrial laboratory for shell effects}

If the observation of $\mathrm{Ni}$ isotopes in terrestrial laboratories spans from ${ }^{48} \mathrm{Ni}$ [35] to ${ }^{78} \mathrm{Ni}$ [36], information about their excitation probability B(E2) was until recently limited to ${ }^{56} \mathrm{Ni}$ and ${ }^{64} \mathrm{Ni}$ on both sides of the valley of stability. From the $2^{+}$-energy pattern of Fig. 6, the sudden rises at $\mathrm{N}=28$ and $\mathrm{N}=40$ indicate the location of shell and subshell closures respectively. In order to estimate their strength, and in particular their rigidity against quadrupole excitations, it is necessary to determine the $\mathrm{B}(\mathrm{E} 2)$ of these nuclei.

The doubly magic nucleus ${ }^{56} \mathrm{Ni}$ was first studied by Kraus et al. [37] with a pp' reaction study and more recently by Yanagisawa et al. [38] by coulomb excitation. The two experiments, though using a different method, converge to a rather large $\mathrm{B}(\mathrm{E} 2)$ of about $600 e^{2} \mathrm{fm}^{4}$. Otsuka et al. [39] and Caurier et al. [40] interpret this large E2 transition strength value as due to large particle-hole $(\mathrm{ph})$ correlations effects in the fp-shell. This effect is enhanced due to the proton-neutron correlations characterizing $\mathrm{N}=\mathrm{Z}$ nuclei [39]. When increasing the number of neutrons from ${ }^{56} \mathrm{Ni}$, the number of particles and holes in the fp-shell above ${ }^{56} \mathrm{Ni}$ is maximum for ${ }^{62} \mathrm{Ni}$. Hence, the $\mathrm{B}(\mathrm{E} 2)$ is increasing up to $900 e^{2} \mathrm{fm}^{4}$ for ${ }^{62} \mathrm{Ni}$, and starts to decrease with the filling of the fp orbitals. Coulomb 


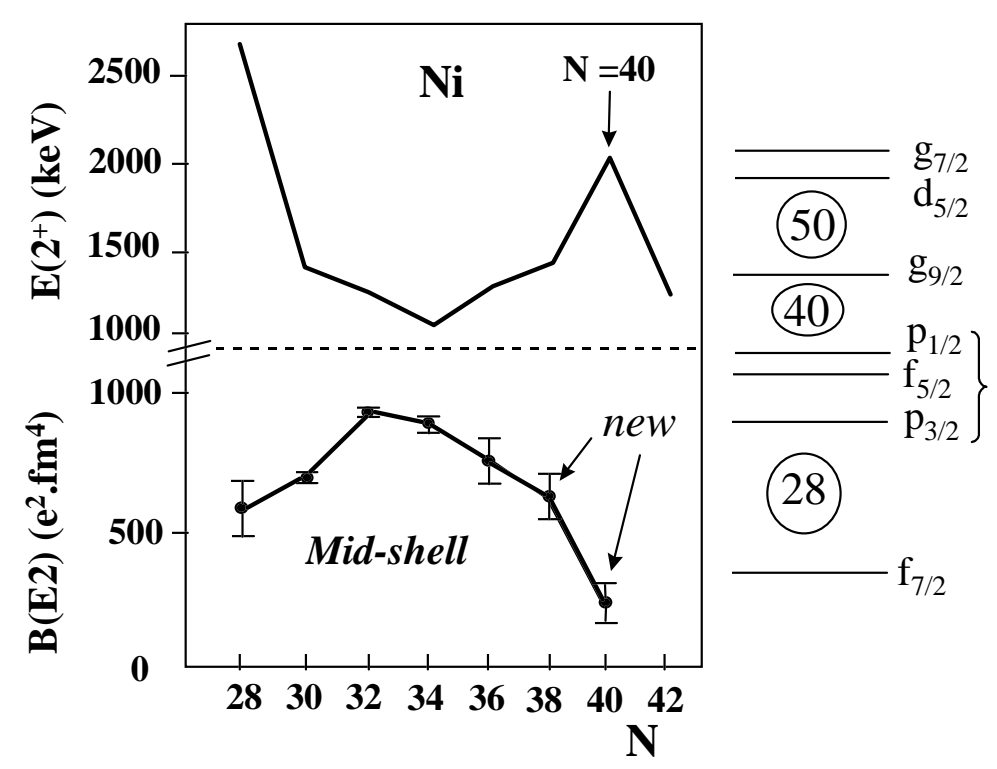

Figure 6. Measured $2^{+}$-energies and B(E2) values as a function of neutron-richness for $\mathrm{Ni}$ isotopes

excitation of ${ }^{66} \mathrm{Ni}$ and ${ }^{68} \mathrm{Ni}$ have been achieved at GANIL via interactions of these secondary beams of about $10^{4} \mathrm{pps}$ with a lead target. Their reduced transition probabilities $B\left(E 2: 0_{1}^{+} \rightarrow 2^{+}\right)$have been measured for the first time [41], showing that the collectivity is strongly decreased at ${ }^{68} N i_{40}$, as compared to other $\mathrm{Ni}$ isotopes. ${ }^{68} \mathrm{Ni}$ has the lowest $\mathrm{B}(\mathrm{E} 2)$ value, $\mathrm{B}(\mathrm{E} 2)=290(70) e^{2} \mathrm{fm}^{4}$, among all hitherto studied $\mathrm{Ni}$ isotopes (Fig. 6 ). This feature can be explained by the subshell closure effect at $\mathrm{N}=40$, characterized by the complete filling of the fp orbitals. Because of the parity change between the fp and gd shells, the $2^{+}$excitation in ${ }^{68} \mathrm{Ni}$ cannot be made by one particle one hole excitation which would provide only negative parity states. The only possibility left is to promote a pair of neutrons from one of the fp orbitals, and to couple it to $2^{+}$into the gd shell. This requires more energy, i.e. $\mathrm{E}\left(2^{+}\right)$increases, and causes a small $\mathrm{B}\left(\mathrm{E} 2 ; 0^{+} \rightarrow 2^{+}\right)$, as this can proceed only via small admixtures. It is interesting to see the rigidity of this subshell closure with respect to the addition of few protons or neutrons to ${ }^{68} \mathrm{Ni}$. In this respect, the determination of the $\mathrm{B}(\mathrm{E} 2)$ of ${ }^{70,72} \mathrm{Ni}$ is already feasible and will probably be determined in a near future. First informations on excited states of ${ }^{70} N i$ has been first provided by isomer decay-studies by Grzywacz et al [42]. From the existence of a $8^{+}$-isomer, the cascade of photons $\left(8^{+} \rightarrow 6^{+} \rightarrow 4^{+} \rightarrow 2^{+} \rightarrow 0^{+}\right)$has been observed and the energies of the levels deduced. The $2^{+}$energy of ${ }^{70} \mathrm{Ni}$ has been found to decrease down to $1.259 \mathrm{MeV}$ as compared to $2.033 \mathrm{MeV}$ [43] in ${ }^{68} \mathrm{Ni}$ and $1.422 \mathrm{MeV}$ in ${ }^{66} \mathrm{Ni}[44]$. The small $2^{+}$energy of ${ }^{66} \mathrm{Fe}[45]$ shows that the removal of two protons from ${ }^{68} \mathrm{Ni}$ already increase the possibility of making protons and neutrons excitations within the fp shell.

Towards $\mathrm{N}=50$ in the $\mathrm{Ni}$ isotopic chain, very few experimental informations is known. The non-observation of the sequence of photons $\left(8^{+} \rightarrow 6^{+} \rightarrow 4^{+} \rightarrow 2^{+} \rightarrow 0^{+}\right)$in ${ }^{72,74} \mathrm{Ni}$ 
as found in ${ }^{68} \mathrm{Ni}[46]$ and ${ }^{70} \mathrm{Ni}[42]$ points towards a change in the structure of Ni nuclei between $\mathrm{N}=40$ and $\mathrm{N}=50$ closed shell. From the decay of ${ }^{70} \mathrm{Co}$, Mueller et al. [47] suggested the presence of deformed configuration in ${ }^{70} \mathrm{Ni}$ at low excitation energy. The mixing of configuration between the spherical g.s. and first deformed minimum may perturb the multiplet configuration and may explain the non observation of the $8^{+}$isomer in ${ }^{70} \mathrm{Ni}$ and ${ }^{72} \mathrm{Ni}$ (see the work of Grawe et al. [48] for a more complete discussion). The recent observation of this $8^{+}$isomer in ${ }^{78} \mathrm{Zn}$ [49], a nucleus very close to ${ }^{78} \mathrm{Ni}$ shows that this $0^{+}, \ldots, 8^{+}$level sequence is re-appearing close to $\mathrm{N}=50$. It might be an indication of a magicity at $\mathrm{N}=50$ though further investigations are necessary to prove it.

\section{CONCLUSIONS}

Tremendous experimental efforts have been achieved in the study of exotic nuclei throughout the world the last few years. The increase of beam intensities and detector efficiency and sensitivity has brought the search of disappearence of magic shell reachable. This review has shown some of the investigations around the neutron numbers $\mathrm{N}=20,28$, and 40. The in-beam spectroscopy method using the projectile fragmentation has provided an innovating method to search for the spectroscopy of nuclei difficult to access by other means. Coulomb excitation of radioactive beams as low as few particle per seconds has been used to determine $\mathrm{E}\left(2^{+}\right)$and $\mathrm{B}(\mathrm{E} 2)$ of even-even nuclei towards or at major closed shells. From these combined studies, and the technique of $\mu$ s delayed isomers, it is possible to draw some conclusions on the $\mathrm{N}=20,28$ and 40 shells. It seems that the $\mathrm{N}=20$ closed shell disappears when increasing the isospin while the $\mathrm{N}=16$ subshell becomes stronger. If confirmed, it is a clear indication of modification of mean field when a large number of neutrons is added to nuclei. In order to put these indications on a more solid footing, experiments have to be extended to ${ }^{24} \mathrm{O},{ }^{27-29} \mathrm{~F}$ and ${ }^{30} \mathrm{Ne}$. Concerning the $\mathrm{N}=28$ closed shell, it is seen from various evidences that the ${ }^{42} S$ is strongly deformed, while ${ }^{43} S$ and ${ }^{44} S$ possibly both exhibit a shape coexistence. The study of Ni istopes has shown that ${ }^{68} \mathrm{Ni}$ has the lowest $\mathrm{B}(\mathrm{E} 2)$ among the known $\mathrm{Ni}$ isotope. It seems however that this "rigidity" against excitations is due to the peculiar nature of the intruder orbital $\mathrm{g}_{9 / 2}$ which lie just above the fp states. The change of parity between $\mathrm{fp}$ and $\mathrm{g}$ states yield to a weak probablity of making quadrupole excitations across $\mathrm{N}=40$, and the high pairing energy in $g_{9 / 2}$ shell is in a large part responsible for the high $2^{+}$energy of ${ }^{68} \mathrm{Ni}$. The recent discovery of the decay of a $\mu$ s isomer in ${ }^{78} \mathrm{Zn}$ has brought new hopes for attaining the doubly magic ${ }^{78} \mathrm{Ni}$.

Acknowledgments: The results discussed in this talk are from experiments performed at GANIL by a large team of physicists from many European laboratories. Both experiments using Coulomb excitation and in-beam gamma spectroscopy with fragmentation reactions benefit from the availability of ${ }^{70} \mathrm{Zn},{ }^{36} \mathrm{~S}$ and ${ }^{48} \mathrm{Ca}$ isotopes kindly provided by our colleagues from DUBNA and from the smooth running of the accelerator from the GANIL crew. The use of the segmented clover detectors was made possible thanks to the EXOGAM collaboration.

I have special acknowledgments to F. Amorini, M. Belleguic, C. Donzaud, M. J. Lopez, S.Leenhardt, D. Sohler, M. Stanoiu and J. Timar who have analyzed the data presented 
in this contribution on Coulomb excitation and in-beam spectroscopy. I also thank F. Azaiez, Z. Dombradi, H. Grawe, F. Nowacki, A. Poves and M. G. Saint-Laurent for valuable discussions.

\section{REFERENCES}

1. J. Dobaczewski et al., Phys. Rev. Lett. 72 (1994) 981.

2. P.-G Reinhard et al., Phys. Rev. C 60 (1999) 014316.

3. C. Thibault et al., Phys. Rev. C 12 (1975) 193.

4. G. Huber et al., Phys. Rev. C 18 (1978) 2342

5. C. Détraz et al., Phys. Rev. C 19 (1978) 171.

6. D. Guillemaud et al., Nucl. Phys. A246(1984)37.

7. R. W. Ibbotson et al., Phys. Lett. B 461 (1998) 2081.

8. T. Motobayashi et al., Phys. Lett. B 346 (1995) 9.

9. B. V. Pritychenko et al., Phys. Lett. B 461 (1999) 322.

10. V. Chisté et al., submitted to PRL.

11. M. Belleguic et al., Phys. Scripta T 88 (2000) 122.

12. F. Azaiez et al. in proceedings of the Int. Conf. "Nuclear Structure 98", Gatlinburg (1998) and F. Azaiez in proceedings of the Int. Conf. "ENPE99", Sevilla, Spain (1999).

13. A. Reed et al., Phys. Rev. C 60 (1999) 24311.

14. O. Tarasov et al., Phys. Lett. B 409 (1997) 64.

15. H. Sakurai et al., Phys. Lett. B 448 (1999)180.

16. P. G. Thirolf et al., Phys. Lett. B 485 (2000) 16.

17. D. Sohler in proceedings of the Exotic nuclear shapes 2000 conference, 15-21 May, Debrecen, Hungary.

18. B. A. Brown predictions from (http://www.nsl.msu.edu/brown/sde.htm)

19. Y. Utsuno et al., Phys. Rev. C 60 (1999) 054315.

20. O. Ozawa et al., Phys. Rev. Lett. 84 (2000) 5493.

21. T. Otsuka, contribution to this conference.

22. W. Nazarewicz, private remark.

23. O. Sorlin et al., Phys. Rev. C 47(1993)2941.

24. O. Sorlin et al., Nucl. Phys. A 583 (1995) 763.

25. P. Möller et al., At. Data and Nucl. Data Tables 66 (1997) 131.

26. F. Sarazin et al., Phys. Rev. Lett. 84 (2000) 5062.

27. O. Sorlin et al., Nucl. Phys. A 669(2000)351.

28. T. R. Werner et al., Phys. Lett. B 335 (1994) 259

29. T. Glasmacher et al., Phys. Lett. B 395 (1997) 163.

30. S. Raman et al., At. Data Nucl. Data Tables 42 (1989) 1

31. R. W. Ibbotson et al., Phys.Rev. C 59 (1999) 642

32. F. Nowacki and E. Caurier, private communication.

33. H. Scheit et al., Phys. Rev. Lett. 77 (1996) 3967.

34. J. Retamosa et al., Phys. Rev. C 55 (1996) 1266.

35. B. Blank et al., Phys. Rev. Lett. 84 (2000) 1116.

36. C. Engelmann et al., Z. Phys. A 352 (1995) 351.

37. G. Kraus et al., Phys. Rev. Lett. 73 (1994) 1773. 
38. Y. Yanagisawa et al., in proc. of the ENAM98 conference, eds B. M. Sherrill et al., p. 610.

39. T. Otsuka et al., Phys. Rev. Lett. 81 (1998) 1588.

40. E. Caurier et al., sumbmitted to Phys. Rev. Lett.

41. S. Leenhardt, thesis work, IPN Orsay and S. Leenhardt et al., Nuovo Cimento, 111A (1998) 733.

42. R. Grzywacz et al., Phys. Rev. Lett. 81 (1998) 766.

43. R. Broda et al., Phys. Rev. Lett. 74 (1995) 868.

44. T. Pawlat et al., Nucl. Phys. A 574 (1994) 623.

45. M. Hannawald et al., Phys. Rev. Lett. 82 (1999) 1391.

46. T. Ishii et al., Phys. Rev. Lett. 84 (2000) 39.

47. W. F. Mueller et al. Phys. Rev. C 61 (2000) 054308

48. H. Grawe et al. in Proc. of the RIKEN Symposium shell Model 2000, Nucl. Phys. A, in print.

49. J. M. Daugas, Phys. Lett. B 476 (2000) 213. 